\title{
Entanglement criteria and full separability of multi-qubit quantum states
}

\author{
Otfried Gühne \\ Institut für Quantenoptik and Quanteninformation, Österreichische Akademie der \\ Wissenschaften, Technikerstr. 21A, 6020 Innsbruck, Austria \\ Institut für Theoretische Physik, Universität Innsbruck, Technikerstr. 25, 6020 Innsbruck, \\ Austria \\ Fachbereich Physik, Universität Siegen, Walter-Flex-Str. 3, 57068 Siegen, Germany
}

\begin{abstract}
We introduce an entanglement criterion to exclude full separability of quantum states. We present numerical evidence that the criterion is necessary and sufficient for the class of GHZ diagonal three-qubit states and estimate the volume of bound entangled states within this class. Finally, we extend our approach to bound entangled states which are not GHZ diagonal.
\end{abstract}

\section{Introduction}

Entanglement is believed to be an important resource in quantum information processing and consequently many works are devoted to its characterization [1, 2]. This characterization becomes complicated, if more than two particles are entangled, since then different classes of entanglement exist. Some methods to distinguish between the different classes have been presented in the literature $3-$ 17]. However, no general solution of the problem is known, not even for specific families of states.

In this paper we present a criterion for the verification of multi-qubit entanglement. The criterion is formulated as a set of simple inequalities for the matrix elements of the state. Our approach is inspired by some existing criteria [12], and also by some recent works on entanglement in the family of graph states [16, 17]. We consider then density matrices which are diagonal in terms of GreenbergerHorne-Zeilinger (GHZ) states and investigate the optimality of our criterion. For that, we derive methods to prove that a given state is separable and it turns out that all GHZ diagonal states under scrutiny are either detected by our criterion or proven to be separable. This allows to estimate the volume of so-called bound entangled states in this class of states. Bound entanglement is a weak form of Preprint submitted to Elsevier

October 12, 2018 
entanglement, where no pure state entanglement can be distilled from; and this phenomenon is central to many open problems in quantum information theory 1]. Finally, we discuss with the help of an example how our ideas can be used to characterize bound entanglement close to the three-qubit W state.

\section{Definitions and statement of the problem}

Before explaining our separability criteria, we introduce the notation and give some examples of existing separability criteria and interesting quantum states.

\subsection{Separability and entanglement}

We consider an $N$-particle system with Hilbert space $\mathcal{H}_{\text {tot }}=\mathcal{H}^{\otimes N}$. Any matrix $\varrho$ acting on $\mathcal{H}_{\text {tot }}$ which is hermitian $\left(\varrho=\varrho^{\dagger}\right)$, has no negative eigenvalues $(\varrho \geq 0)$ and is normalized $[\operatorname{Tr}(\varrho)=1]$ is a valid density matrix of some quantum state. By definition, a state is fully separable, if it can be written as a convex combination of product states,

$$
\varrho=\sum_{k} p_{k}\left|a_{k}\right\rangle\left\langle a_{k}|\otimes| b_{k}\right\rangle\left\langle b_{k}|\otimes \ldots \otimes| z_{k}\right\rangle\left\langle z_{k}\right|,
$$

where the $p_{k}$ are non-negative $\left(p_{k} \geq 0\right)$ and normalized $\left(\sum_{k} p_{k}=1\right)$; in other words, they form a probability distribution. If a state cannot be written as in Eq. (1) it is entangled in some sense. In general, it is not easy to check whether a given quantum state is fully separable or not, see Refs. 3-13] for some existing sufficient criteria for entanglement and Refs. [18, 19] for numerical tests to prove separability. Especially if a state is only weakly entangled, proving entanglement is not straightforward.

In this paper we will derive criteria, which allow to prove that a state is not fully separable and hence contains some entanglement. It should be stressed that there are more refined notions of entanglement for multiparticle systems (e.g., genuine multipartite entanglement) which can be more relevant for special situations (e.g., experiments). We will, however, concentrate on full separability as the basic definition, for a discussion of recent results on the other classifications see the reviews in Refs. [1, 2]. Moreover, we will focus our discussion on the case of three qubits, but our results can directly be generalized to more particles.

\subsection{A criterion for full separability in terms of the matrix elements}

Let us explain a separability criterion for full separability derived in Ref. [12], which we will generalize later. For three qubits, consider the $8 \times 8$-density matrix with entries $\varrho_{i, j}$. Here and in the following, we always order the basis vectors in the canonical way, $\{|000\rangle,|001\rangle,|010\rangle, \ldots,|111\rangle\}$. Then, if the state is fully separable, the entries fulfill

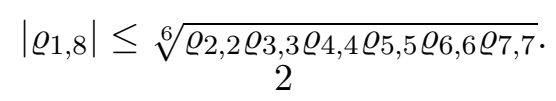


The idea of the proof is as follows: It is easy to check that for a pure fully separable state equality holds in Eq. (2). Then, $\left|\varrho_{1,8}\right|$ is convex in $\varrho$ [a function $f(\varrho)$ is convex if $\left.f\left[p \varrho_{1}+(1-p) \varrho_{2}\right] \leq p f\left(\varrho_{1}\right)+(1-p) f\left(\varrho_{2}\right)\right]$, while the right-hand side of Eq. (2) is concave [i.e., $\left.f\left[p \varrho_{1}+(1-p) \varrho_{2}\right] \geq p f\left(\varrho_{1}\right)+(1-p) f\left(\varrho_{2}\right)\right]$. This implies the bound for mixed fully separable states.

The right-hand side of the inequality (2) may be replaced by other expressions, for instance, $\left|\varrho_{1,8}\right| \leq \sqrt[4]{\varrho_{2,2} \varrho_{3,3} \varrho_{5,5} \varrho_{8,8}}$ holds for separable states. Then, this criterion is able to detect the entanglement in a family of states which are separable with respect to any bipartition, but not fully separable [12]. This is then a bound entangled state and the criterion improves existing criteria for this family significantly.

In the following, we will extend the criterion in Eq. (2) by considering more than one off-diagonal element on the left-hand side. We will also discuss the optimal choice of the right-hand side.

\subsection{A bound entangled state}

Let us now introduce a bound entangled state which motivates our approach. Consider the family of three-qubit density matrices introduced by A. Kay [16],

$$
\varrho_{\mathrm{AK}}(\hat{\alpha})=\frac{1}{8+8 \hat{\alpha}}\left(\begin{array}{cccccccc}
4+\hat{\alpha} & 0 & 0 & 0 & 0 & 0 & 0 & 2 \\
0 & \hat{\alpha} & 0 & 0 & 0 & 0 & 2 & 0 \\
0 & 0 & \hat{\alpha} & 0 & 0 & -2 & 0 & 0 \\
0 & 0 & 0 & \hat{\alpha} & 2 & 0 & 0 & 0 \\
0 & 0 & 0 & 2 & \hat{\alpha} & 0 & 0 & 0 \\
0 & 0 & -2 & 0 & 0 & \hat{\alpha} & 0 & 0 \\
0 & 2 & 0 & 0 & 0 & 0 & \hat{\alpha} & 0 \\
2 & 0 & 0 & 0 & 0 & 0 & 0 & 4+\hat{\alpha}
\end{array}\right) .
$$

This matrix is a valid quantum state for $\hat{\alpha} \geq 2$ and it is separable for $\hat{\alpha} \geq$ $2 \sqrt{2}$. This has been proved in Ref. [16] by writing down an explicit separable decomposition as in Eq. (11). Furthermore, using the algorithm outlined in Ref. [5] it was shown numerically that the state is entangled for $2 \leq \hat{\alpha} \leq 2.828$. Note that for any $\hat{\alpha} \geq 2$ the state has a positive partial transpose (PPT) for any bipartition. This does not only imply that no entanglement can be distilled from it 1 ; moreover, since the state is diagonal in the GHZ basis (see also below), it also implies that

\footnotetext{
${ }^{1}$ At this point, one should mention that undistillable entanglement can arise in multiparticle systems in a simple way: If $\varrho$ is entangled with respect to one bipartition, but PPT with respect to another bipartition, this implies already that $\varrho$ is entangled, but multipartite undistillable. In this paper, however, we use a more restrictive definition: we consider bound entangled states, that are separable for any bipartition, but not fully separable. This means that they are undistillable, even if arbitrary parties join.
} 
the state is separable for all bipartitions. Namely, from the fact that a twoqubit Bell diagonal state with a positive partial transpose is separable, one can conclude that a GHZ diagonal state that has a positive partial transposition is also separable for that partition [20].

The state in Eq. (3) is an example of a GHZ diagonal state. These are of the form

$$
\varrho=\sum_{k=1}^{8} p_{k}\left|G H Z_{k}\right\rangle\left\langle G H Z_{k}\right|,
$$

where the GHZ state basis consists of the eight vectors $\left|G H Z_{k}\right\rangle=\left|0 x_{2} x_{3}\right\rangle \pm$ $\left|1 \bar{x}_{2} \bar{x}_{3}\right\rangle$ where $x_{i}, \bar{x}_{i} \in\{0,1\}$ and $x_{i} \neq \bar{x}_{i}$. Alternatively, the GHZ diagonal states can be written as

$\varrho=\frac{1}{8}\left[\mathbb{1}+\lambda_{2} Z Z \mathbb{1}+\lambda_{3} Z \mathbb{1} Z+\lambda_{4} \mathbb{1} Z Z+\lambda_{5} X X X+\lambda_{6} Y Y X+\lambda_{7} Y X Y+\lambda_{8} X Y Y\right]$,

where $X, Y$, and $Z$ denote the the Pauli matrices and tensor product symbols have been omitted. The observables occurring in Eq. (5) are the so-called stabilizing observables of the GHZ states, see Ref. 21] for a further discussion.

GHZ diagonal states have been intensively discussed in the literature before $[3,12,20,22]$ and are interesting from several perspectives: They have a simple structure, since only the diagonal and the anti-diagonal elements of the matrix can be nonzero, and they occur naturally in certain types of decoherence processes [23]. Furthermore, any state can be transformed to a GHZ diagonal state (without changing the fidelities of the GHZ states) by local operations [3, 21]. This means that if the remaining GHZ diagonal state is entangled, the initial state must have been entangled, too. On the other hand, if one has a GHZ diagonal state $\varrho_{1}$ and a second separable state $\varrho_{2}$ which is mapped to $\varrho_{1}$ by these local operations, then $\varrho_{1}$ must be separable, too.

The state $\varrho_{\mathrm{AK}}$ is not detected by the criterion in Eq. (2) or variants thereof. The reason lies in the fact that Eq. (2) considers only single offdiagonal elements, and neglects the (phase) relations between them. It is the main purpose of this paper to develop an improvement of Eq. (2) which takes into account all offdiagonal elements at the same time. This will finally prove analytically that the state $\varrho_{\mathrm{AK}}$ is entangled if $2 \leq \hat{\alpha}<2 \sqrt{2}$.

\section{The separability criterion}

In this section we will now formulate the separability criterion. We will restrict our attention to three qubits, but it should be stressed that our approach can straightforwardly be generalized to an arbitrary number of qubits.

To start, consider a pure product state,

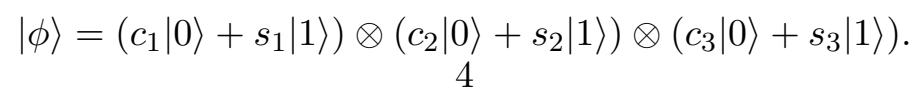


Here, the complex coefficients $c_{i}$ and $s_{i}$ fulfill the normalization $\left|c_{i}\right|^{2}+\left|s_{i}\right|^{2}=1$. Let us consider the corresponding $8 \times 8$ density matrix $\varrho=|\phi\rangle\langle\phi|$. The offdiagonal elements can be written as

$$
\begin{aligned}
& \varrho_{1,8}=c_{1} c_{2} c_{3} s_{1}^{*} s_{2}^{*} s_{3}^{*}=\kappa e^{i(a+b+c),} \\
& \varrho_{2,7}=c_{1} c_{2} s_{3} s_{1}^{*} s_{2}^{*} c_{3}^{*}=\kappa e^{i a}, \\
& \varrho_{3,6}=c_{1} s_{2} c_{3} s_{1}^{*} c_{2}^{*} s_{3}^{*}=\kappa e^{i b}, \\
& \varrho_{5,4}=s_{1} c_{2} c_{3} c_{1}^{*} s_{2}^{*} s_{3}^{*}=\kappa e^{i c} .
\end{aligned}
$$

Here, we used the notations $a=\phi_{1}+\phi_{2}-\phi_{3}, b=\phi_{1}-\phi_{2}+\phi_{3}$, and $c=$ $-\phi_{1}+\phi_{2}+\phi_{3}$, where the phases are defined via $c_{k} s_{k}^{*}=\left|c_{k} s_{k}\right| e^{i \phi_{k}}$. Furthermore, we set $\kappa=\left|c_{1} s_{1}\right|\left|c_{2} s_{2}\right|\left|c_{3} s_{3}\right|$. Note that the other offdiagonal elements follow from these and the fact that $\varrho$ is hermitian.

From Eq. (17) one can conclude two things. First, for a product states the absolute value of all the offdiagonal elements is the same. Second, the four phases are not arbitrary; they depend only on three parameters.

To proceed, consider a linear functional like

$$
\mathcal{L}(\varrho, \vec{X})=\Re\left(X_{1} \varrho_{1,8}+X_{2} \varrho_{2,7}+X_{3} \varrho_{3,6}+X_{4} \varrho_{5,4}\right),
$$

where $\vec{X}=\left(X_{1}, X_{2}, X_{3}, X_{4}\right)$ is a vector of complex coefficients and $\Re(\ldots)$ denotes the real part. The functional $\mathcal{L}$ is compatible with convex combinations of the quantum state, i.e., one has $\mathcal{L}\left[p \varrho_{1}+(1-p) \varrho_{2}\right]=p \mathcal{L}\left(\varrho_{1}\right)+(1-p) \mathcal{L}\left(\varrho_{2}\right)$. For a pure separable state, $\mathcal{L}$ is given by

$$
\begin{aligned}
\mathcal{L}(|\phi\rangle)= & \kappa \mathcal{F}(\vec{X}) \text { with } \\
\mathcal{F}(\vec{X})= & \Re\left(X_{1}\right) \cos (a+b+c)-\Im\left(X_{1}\right) \sin (a+b+c)+\Re\left(X_{2}\right) \cos (a) \\
& -\Im\left(X_{2}\right) \sin (a)+\Re\left(X_{3}\right) \cos (b)-\Im\left(X_{3}\right) \sin (b) \\
& +\Re\left(X_{4}\right) \cos (c)-\Im\left(X_{4}\right) \sin (c) .
\end{aligned}
$$

In order to obtain an extension of the separability condition in Eq. (2) we need two more facts. First, for given coefficients $\vec{X}$ one may compute the maximum given by

$$
C(\vec{X})=\sup _{a, b, c}|\mathcal{F}(\vec{X})|
$$

For given values of $\vec{X}$ this can usually be computed analytically (see also below) or with a simple numerical optimization.

Second, we have to characterize $\kappa$ for product states. For them we have

$$
\begin{aligned}
& \kappa=\sqrt[4]{\varrho_{1,1} \varrho_{4,4} \varrho_{6,6} \varrho_{7,7}}=\sqrt[4]{\varrho_{2,2} \varrho_{3,3} \varrho_{5,5} \varrho_{8,8}} \\
&=\sqrt{\varrho_{k, k} \varrho_{9-k, 9-k}} \text { for all } 1 \leq k \leq 4 . \\
& 5
\end{aligned}
$$


There are further equalities of this type (e.g., $\kappa=\sqrt[6]{\varrho_{2,2} \varrho_{3,3} \varrho_{4,4} \varrho_{5,5} \varrho_{6,6} \varrho_{7,7}}$ ) and in view of Eq. (2) one might be tempted to use them. This, however, will not give stronger criteria: $\kappa$ will be used to deliver an upper bound, and in general a bound like $x \leq \sqrt[3]{\alpha \beta \gamma}$ [corresponding to Eq. (2)] is weaker than the bound $x \leq \min \{\alpha, \beta, \gamma\}$ [corresponding to the last line in Eq. (11)]. In a similar way one can directly see that other possible bounds (as used in Ref. [12]) can be derived from the terms in Eq. (11).

Putting it all together, we can formulate:

Observation. Let $\vec{X}$ be some coefficients and $C(\vec{X})$ be defined as in Eq. (10). Then, if $\varrho$ is fully separable the inequality

$$
\begin{aligned}
|\mathcal{L}(\varrho, \vec{X})| \leq & C(\vec{X}) \min \left\{\sqrt[4]{\varrho_{1,1} \varrho_{4,4} \varrho_{6,6} \varrho_{7,7}}, \sqrt[4]{\varrho_{2,2} \varrho_{3,3} \varrho_{5,5} \varrho_{8,8}},\right. \\
& \left.\sqrt{\varrho_{k, k} \varrho_{9-k, 9-k}}(\text { with } 1 \leq k \leq 4)\right\}
\end{aligned}
$$

holds and violation of this inequality implies entanglement.

To prove this criterion, note first that Eq. (12) holds for pure product states. Furthermore, the left-hand side is a convex function of the state, while the righthand side is concave [12]. Since a mixed fully separable state is a convex combination of fully separable pure states, Eq. (12) has to hold.

It remains to discuss which parameters $\vec{X}$ should be chosen in order to detect a given state $\varrho$. A simple choice is $\vec{X} \sim\left(\varrho_{1,8}^{*}, \varrho_{2,7}^{*}, \varrho_{3,6}^{*}, \varrho_{5,4}^{*}\right)$, since then the Cauchy-Schwarz inequality guarantees that $\mathcal{L}(\varrho, \vec{X})$ is maximal for all $\vec{X}$ with the same normalization. The optimal choice, however, is to choose $\vec{X}$ such that $|\mathcal{L}(\varrho, \vec{X})| / C(\vec{X})$ is maximal.

\section{Examples}

\subsection{The bound entangled state from Eq. (3)}

As a first example, let us discuss the state $\varrho_{\text {AK }}$ from Eq. (3). First, let us take a look at

$$
C[\vec{X}=(\delta, \alpha, \beta, \gamma)]=\sup _{a, b, c}[\delta \cos (a+b+c)+\alpha \cos (a)+\beta \cos (b)+\gamma \cos (c)],
$$

where the coefficients $\delta, \alpha, \beta, \gamma$ are real. This case is important for GHZ-diagonal states, since for them all anti-diagonal elements are real.

If these coefficients are all positive, it is clear that the maximum is $C=|\delta|+$ $|\alpha|+|\beta|+|\gamma|$ and is attained at $a=b=c=0$. The same value for $C$ is obtained, if two or four of the coefficients are negative, because with transformations like $a \mapsto a+\pi$ one can flip two arbitrary signs of the cosine terms. 
If one of the coefficients is negative (we choose $\gamma<0$ for definiteness) one finds after some algebra that the optimum can be attained at

$$
\begin{array}{ll}
\sin (a)=\frac{1}{2 \alpha} \frac{\sqrt{Q}}{\sqrt{R}}, & \cos (a)= \pm \sqrt{1-\sin (a)^{2}} \\
\sin (b)=\frac{1}{2 \beta} \frac{\sqrt{Q}}{\sqrt{R}}, & \cos (b)= \pm \sqrt{1-\sin (b)^{2}} \\
\sin (c)=\frac{1}{2 \gamma} \frac{\sqrt{Q}}{\sqrt{R}}, & \cos (c)= \pm \sqrt{1-\sin (c)^{2}}
\end{array}
$$

with

$$
\begin{aligned}
Q= & -(\alpha \beta \delta+\alpha \beta \gamma-\alpha \delta \gamma-\beta \delta \gamma)(\alpha \beta \delta-\alpha \beta \gamma+\alpha \delta \gamma-\beta \delta \gamma) \\
& (\alpha \beta \delta-\alpha \beta \gamma-\alpha \delta \gamma+\beta \delta \gamma)(\alpha \beta \delta+\alpha \beta \gamma+\alpha \delta \gamma+\beta \delta \gamma), \\
R= & \alpha \beta \gamma \delta(\alpha \beta-\delta \gamma)(\alpha \gamma-\beta \delta)(\alpha \delta-\beta \gamma) .
\end{aligned}
$$

This is the optimal solution, if the $\sin (\ldots)$ are real and their absolute value is not larger than one. Furthermore, one has to distribute maximally one negative sign in the $\cos (\ldots)$ terms such that the condition $\delta \sin (a+b+c)+\alpha \sin (a)=0$ (originating from setting the derivative to zero) holds. If the conditions on $\sin (\ldots)$ are not met, the optimum is just given by a choice of $\{a, b, c\} \in\{0, \pi\}$ resulting in $C=\alpha+\beta+\gamma+\delta$ or $C=\alpha+\beta-\gamma-\delta$ etc. The solution is of the same structure, if three of the coefficients $\alpha, \beta, \gamma, \delta$ are negative.

Applying this to the state from Eq. (3) one finds that $C[\vec{X}=(1,1,-1,1)]=$ $2 \sqrt{2}$. According to Eq. (12), the state is entangled, if $8>2 \sqrt{2} \hat{\alpha} \Leftrightarrow \hat{\alpha}<2 \sqrt{2}$, which proves the numerical result. Recall that for larger values of $\hat{\alpha}$ the state can be proven to be separable (see Ref. [16] and also below), so the criterion is optimal.

\subsection{Random GHZ diagonal states}

As a second example, we consider randomly chosen GHZ diagonal states [see also Eqs. (4. 5)]. Here, we also want to estimate how good the criteria are, in the sense that we want to test whether some entangled states escape from the detection 2

Consequently, we need a method to prove that a given state is separable. This is, in general, not straightforward, but there are two possibilities that we used: First, in Ref. [19] a simple iterative algorithm was proposed that can be used to prove different forms of separability for general quantum states.

Second, for the special case of GHZ diagonal states one can also use the ideas proposed recently in Refs. [16, 17]. Let us explain them step by step. First, a state

\footnotetext{
${ }^{2}$ For results on certain subclasses of GHZ diagonal states see Refs. [16, 17, 22].
} 
like $\varrho \sim \alpha \mathbb{1}+\alpha Z Z \mathbb{1}$ is separable, as it is diagonal in a product basis and has no negative eigenvalues. Then, consider an operator $\mathcal{A}=\lambda_{2} Z Z \mathbb{1}+\lambda_{3} Z \mathbb{1} Z+\lambda_{4} \mathbb{1} Z Z$. The minimal eigenvalue of it is given by $\lambda_{-}=\min \left\{\lambda_{2}+\lambda_{3}+\lambda_{4}, \lambda_{2}-\lambda_{3}-\right.$ $\left.\lambda_{4},-\lambda_{2}+\lambda_{3}-\lambda_{4},-\lambda_{2}-\lambda_{3}+\lambda_{4}\right\}$ and $\lambda_{-}$is never positive. Therefore, a state like $\varrho \sim\left|\lambda_{-}\right| \mathbb{1}+\mathcal{A}$ is also separable, as it is diagonal in the computational basis and positive semidefinite.

Considering the state in Eq. (5), this allows already to conclude that a GHZ diagonal state is separable, if $\left|\lambda_{-}\right|+\left|\lambda_{5}\right|+\left|\lambda_{6}\right|+\left|\lambda_{7}\right|+\left|\lambda_{8}\right| \leq 1$. However, inspired by Ref. [17] we can go further: taking the operator $\mathcal{B}=\lambda_{5} X X X+\lambda_{6} Y Y X+$ $\lambda_{7} Y X Y+\lambda_{8} X Y Y$ we may try to rewrite it as

$$
\mathcal{B}=\frac{1}{2}\left[\left(A_{1}+B_{1}\right) \otimes\left(A_{2}+B_{2}\right) \otimes\left(A_{3}+B_{3}\right)+\left(A_{1}-B_{1}\right) \otimes\left(A_{2}-B_{2}\right) \otimes\left(A_{3}-B_{3}\right)\right],
$$

with $A_{i}=\mu \cos \left(\vartheta_{i}\right) X$ and $B_{i}=\mu \sin \left(\vartheta_{i}\right) Y$. Then, the minimal eigenvalue of each of the terms in the right-hand side of Eq. (16) is given by $-\mu^{3}$, and the matrix $\varrho \sim \mu^{3} \mathbb{1}+\mathcal{B}$ is separable.

If all $\lambda_{i}$ in $\mathcal{B}$ are positive, a solution of Eq. (16) can be found with

$$
\mu^{3}\left(\lambda_{5}, \lambda_{6}, \lambda_{7}, \lambda_{8}\right)=\frac{\sqrt{\left(\lambda_{5} \lambda_{6}+\lambda_{7} \lambda_{8}\right)\left(\lambda_{5} \lambda_{7}+\lambda_{6} \lambda_{8}\right)\left(\lambda_{5} \lambda_{8}+\lambda_{6} \lambda_{7}\right)}}{\sqrt{\lambda_{5} \lambda_{6} \lambda_{7} \lambda_{8}}}
$$

and the same solution holds if an even number of $\lambda_{i}$ are negative. Therefore, in this case the state is separable, if $\left|\lambda_{-}\right|+\mu^{3} \leq 1$. For the case that an odd number of the $\lambda_{i}$ in $\mathcal{B}$ is negative, a decomposition as in Eq. (16) will lead to nonhermitian $A_{i}$ and $B_{i}$ which does not help. Then, only the separability condition $\left|\lambda_{-}\right|+\left|\lambda_{5}\right|+\left|\lambda_{6}\right|+\left|\lambda_{7}\right|+\left|\lambda_{8}\right| \leq 1$ remains. But the case that $\prod_{i=5}^{8} \lambda_{i}<0$ is exactly the case, for which it has been shown already in Ref. [16] that the PPT criterion is necessary and sufficient for full separability (note the different sign conventions in Ref. [16]).

In some cases, these conditions can still be improved. For that, one may consider operators of the form

$\mathcal{X}=p\left(A_{1}+B_{1}\right) \otimes\left(A_{2}+B_{2}\right) \otimes\left(A_{3}+B_{3}\right)+(1-p)\left(\hat{A}_{1}-\hat{B}_{1}\right) \otimes\left(\hat{A}_{2}-\hat{B}_{2}\right) \otimes\left(\hat{A}_{3}-\hat{B}_{3}\right)$

with $A_{i}=\alpha_{i} X, B_{i}=\beta_{i} Y, \hat{A}_{i}=\hat{\alpha}_{i} X, \hat{B}_{i}=\hat{\beta}_{i} Y$, and $0 \leq p \leq 1$. The minimal eigenvalues of the two terms are $\eta=-\prod_{i}\left(\sqrt{\alpha_{i}^{2}+\beta_{i}^{2}}\right)$ and $\hat{\eta}=-\prod_{i}\left(\sqrt{\hat{\alpha}_{i}^{2}+\hat{\beta}_{i}^{2}}\right)$, respectively. Therefore, the state

$$
\varrho_{\text {sep }} \sim(p|\eta|+(1-p)|\hat{\eta}|) \mathbb{1}+\mathcal{X}
$$

is separable.

This state $\varrho_{\text {sep }}$ is not diagonal in the GHZ basis as it contains also terms like $X X Y$ etc. But, by applying local operations one can make it GHZ diagonal 


\begin{tabular}{|l|c||l|r|}
\hline $\begin{array}{l}\text { Entangled } \\
\text { [via Eq. (12) })\end{array}$ & $91.32 \%$ & NPT for some partition & $90.61 \%$ \\
\cline { 3 - 4 } & & PPT, but violating Eq. (12) & $0.71 \%$ \\
\hline Separable & \multirow{2}{*}{$8.68 \%$} & Via Eqs. (16, 17) & $8.41 \%$ \\
\cline { 3 - 4 } & & Using in addition Eqs. (18, 19) & $0.27 \%$ \\
\hline \hline Total & $100 \%$ & & $100 \%$ \\
\hline
\end{tabular}

Table 1: Fractions of the randomly generated states, which are detected by the different criteria. See the text for details.

without changing the weights of $X X X, X Y Y, Y X Y$, and $Y Y X$. In fact, after making the terms in Eq. $18 \mathrm{GHZ}$ diagonal, a convex combination of two times Eq. 16 arises.

Consequently, if we consider a given GHZ diagonal state $\varrho_{\mathrm{ghz}}=\chi \mathbb{1}+\mathcal{B}$ and find a separable state like $\varrho_{\text {sep }}$ with the same weights [that is, $p \alpha_{1} \alpha_{2} \alpha_{3}+(1-$ $p) \hat{\alpha}_{1} \hat{\alpha}_{2} \hat{\alpha}_{3}=\lambda_{5}$ etc.] then $\varrho_{\mathrm{ghz}}$ must be separable if $\chi \geq[p|\eta|+(1-p)|\hat{\eta}|]$. The search for the appropriate $\mathcal{X}$ can easily be done numerically. This criterion is stronger than the one of Eqs. (16, 17) since it contains the latter as the special case $p=1 / 2$.

To test our criteria, we have generated $10^{6} \mathrm{GHZ}$ diagonal states, by choosing the eigenvalues $p_{k}$ in Eq. (4) randomly from the seven-dimensional simplex in $\mathbb{R}^{8}$. Then, we tested the criterion of the positivity of the partial transposition [1, 2] as well as our new criterion from Eq. (12). For this criterion we found the optimal $\vec{X}$ via a simple numerical optimization. Note that for GHZ diagonal states the criterion in Eq. (12) is strictly stronger than the PPT criterion: For instance, a state that is PPT with respect to the $A \mid B C$ partition has to fulfill $\left|\varrho_{18}\right| \leq \sqrt{\varrho_{44} \varrho_{55}}$ which is a special case of Eq. (12). For the states that were not detected by these criteria, we have tried to prove that they are separable using the ideas from above. The results are given in Table I.

First, it is important that any state which was not detected by the new criterion in Eq. (12) was proven to be separable. This gives clear evidence that the criterion is a necessary and sufficient entanglement criterion for GHZ diagonal states. Moreover, the volume of the bound entangled states among the GHZ diagonal states can be estimated to be $0.7 \%$.

Finally, we also compared the general separability algorithm from Ref. [19] with the special criteria for GHZ diagonal states in Eqs. (16-19). It turns out that the general algorithm succeeds for $96 \%$ of the separable states and is therefore a powerful tool to prove separability, which can also be used if a state is not GHZ diagonal. 


\section{Bound entanglement in the vicinity of the $\mathrm{W}$ state}

The previous criterion was well suited for GHZ diagonal states. For other states, one may first apply local transformations in order to bring them close to a GHZ diagonal state. Nevertheless, this will not always succeed and one can therefore ask whether similar ideas can be used for other bound entangled states, which are not GHZ diagonal. To see that this is possible, let us consider the state investigated by P. Hyllus [24],

$$
\varrho_{\mathrm{PH}}(\eta)=\frac{1}{3+2 \eta+3 / \eta}\left(\begin{array}{cccccccc}
2 \eta & 0 & 0 & 0 & 0 & 0 & 0 & 0 \\
0 & 1 & 1 & 0 & 1 & 0 & 0 & 0 \\
0 & 1 & 1 & 0 & 1 & 0 & 0 & 0 \\
0 & 0 & 0 & 1 / \eta & 0 & 0 & 0 & 0 \\
0 & 1 & 1 & 0 & 1 & 0 & 0 & 0 \\
0 & 0 & 0 & 0 & 0 & 1 / \eta & 0 & 0 \\
0 & 0 & 0 & 0 & 0 & 0 & 1 / \eta & 0 \\
0 & 0 & 0 & 0 & 0 & 0 & 0 & 0
\end{array}\right) .
$$

This state is close to the three-qubit W state $|W\rangle=(|001\rangle+|010\rangle+|100\rangle) / \sqrt{3}$, and is separable with respect to any bipartition, but not fully separable. The entanglement in this state is not detected by the methods presented in Ref. [12].

To investigate the entanglement properties of these states, consider first filter operations of the form

$$
\mathcal{F}_{\text {tot }}=\mathcal{F} \otimes \mathcal{F} \otimes \mathcal{F} \text { with } \mathcal{F}=\left(\begin{array}{cc}
\frac{1}{x} & 0 \\
0 & x^{2}
\end{array}\right)
$$

Under this filtering operation the state transforms like $\varrho_{\mathrm{PH}}(\eta) \mapsto \mathcal{F}_{\text {tot }}\left[\varrho_{\mathrm{PH}}(\eta)\right] \mathcal{F}_{\text {tot }}$ $\sim \varrho_{\mathrm{PH}}\left(\eta / x^{6}\right)$. Therefore, all states in this family share the same entanglement properties and one may focus on the case that $\eta=\sqrt{3 / 2}$, where the off-diagonal terms are maximal, $\left|\varrho_{2,3}\right|+\left|\varrho_{3,5}\right|+\left|\varrho_{5,2}\right|=1 /(1+\sqrt{8 / 3}) \approx 0.38$.

In order to derive a separability criterion, we consider a pure product state as in Eq. (66) with $\left|s_{1}\right|^{2}\left|s_{2}\right|^{2}\left|s_{3}\right|^{2}=\varrho_{8,8} \leq \varepsilon$. Then, for some $i \in\{1,2,3\}$ the bound $\left|s_{i}\right| \leq \sqrt[6]{\varepsilon}$ must hold, and from that it follows after a short calculation (using $\left.\left|c_{j}\right| \leq 1\right)$ that $\left|\varrho_{2,3}\right|+\left|\varrho_{3,5}\right|+\left|\varrho_{5,2}\right| \leq \sqrt[6]{\varepsilon}+1 / 4$. Therefore, any pure separable state obeys

$$
\left|\varrho_{2,3}\right|+\left|\varrho_{3,5}\right|+\left|\varrho_{5,2}\right| \leq \sqrt[6]{\varrho_{8,8}}+1 / 4 .
$$

The left-hand side of this inequality is convex, while the right-hand side is concave, so this inequality holds also for mixed separable states. The state $\varrho_{\mathrm{PH}}$ with $\eta=\sqrt{3 / 2}$ clearly violates it (also if some noise is added), so the states in the family $\varrho_{\mathrm{PH}}(\eta)$ must be entangled. 


\section{Conclusion}

In conclusion, we have presented a criterion for full separability in terms of inequalities for the density matrix elements. The criterion seems to be necessary and sufficient for GHZ diagonal three-qubit states and allows to characterize bound entangled states. Finally, we showed that our ideas can also be extended to different families of bound entangled states close to the $\mathrm{W}$ state.

There are several possibilities to extend our work in the future. First, an analytical proof of the necessity of the criterion for GHZ diagonal states would be desireable. Then, an explicit investigation of the criteria for four or more qubits would be of interest. Finally, one could try to extend our analysis to the more general class of states which are diagonal in a graph state basis.

We thank B. Jungnitsch, P. Hyllus, A. Kay, M. Kleinmann, T. Moroder, S. Niekamp and M. Seevinck for discussions. This work has been supported by the FWF (START Prize and SFB FOQUS) and the EU (NAMEQUAM).

\section{References}

[1] R. Horodecki et al., Rev. Mod. Phys. 81, 865 (2009).

[2] O. Gühne and G. Tóth, Phys. Reports 474, 1 (2009).

[3] W. Dür and I. Cirac, Phys. Rev. A 61, 042314 (2000).

[4] M. Horodecki, P. Horodecki, and R. Horodecki, Phys. Lett. A 283, 1 (2000).

[5] A.C. Doherty, P.A. Parrilo, and F.M. Spedalieri, Phys. Rev. A 71, 032333 (2005).

[6] C.S. Yu and H.S. Song, Phys. Rev. A 72, 022333 (2005).

[7] P. Wocjan and M. Horodecki, Open Syst. Inf. Dyn. 12, 331 (2005).

[8] L. Clarisse and P. Wocjan, Quantum Inf. Comput. 6, 277 (2006).

[9] A. Hassan and P. Joag, Quantum Inf. Comput. 8, 9 (2008).

[10] P. Badziąg et al., Phys. Rev. Lett. 100, 140403 (2008).

[11] G. Tóth et al., Phys. Rev. A 79, 042334 (2009).

[12] O. Gühne and M. Seevinck, New J. Phys. 12, 053002 (2010).

[13] M. Huber et al., Phys. Rev. Lett. 104, 210501 (2010).

[14] S. Niekamp, M. Kleinmann, and O. Gühne Phys. Rev. A 82, 022322 (2010).

[15] O. Gittsovich, P. Hyllus, and O. Gühne, Phys. Rev. A 82, 032306 (2010).

[16] A. Kay, arXiv:1006.5197.

[17] A. Kay, J. Phys. A: Math. Theor. 43, 495301 (2010).

[18] M. Navascues, M. Owari, and M.B. Plenio, Phys. Rev. Lett. 103, 160404 (2009).

[19] J.T. Barreiro et al., Nature Physics 6, 943 (2010).

[20] K. Nagata, Int. J. Theor. Phys. 48, 3358 (2009).

[21] M. Hein et al., quant-ph/0602096.

[22] A.O. Pittenger and M.H. Rubin, Phys. Rev. A 62, 042306 (2000).

[23] L. Aolita et al., Phys. Rev. Lett. 100, 080501 (2008).

[24] P. Hyllus, Witnessing entanglement in qudit systems, PhD thesis, University of Hannover (2005). 